\title{
REVIEW \\ THE PROSPECTS FOR GENETIC CONTROL OF FILARIASIS VECTORS ${ }^{1)}$
}

\author{
K. S. RAI \\ Department of Biology, University of Notre Dame \\ Notre Dame, Indiana 46556
}

Received November 30, 1970

\section{INTRODUCTION}

Considerable progress has been made during recent years in the field of insect pest control through genetic manipulation of populations. As a result, genetic control mechanisms are currently being developed for a number of important pest species. In view of the environmental contamination that ensues from the use of insecticidal chemicals and the fact that insect populations rapidly become resistant to most insecticides, it is imperative that alternate control methods be developed and field tested.

As is well known, filariasis constitutes one of the most important public health problems on a global scale. Because of the fact that improvements in sanitary and public health facilities often do not keep pace with urbanization, the incidence of filariasis in several countries may be on the increase. Furthermore, areas which were once uninhabited and therefore free of filariasis, show relatively high incidence as they develop into urban localities (Rai 1966,1969a). This undoubtedly happens because the most important filariasis vector, Culex pipiens complex thrives in polluted conditions brought about by poor sanitation.

Although several species of mosquitoes have been implicated as vectors of filariasis in different countries, including Japan, the primary Japanese vectors are members of the Culex pipiens complex, particularly C. p. pallens and Aedes togoi. In view of the observations of Bekku (1956) indicating a continuous variation in $C$. pipiens populations in Japan as between $C$. p. pipiens in the north and $C$. p. fatigans in the south, $C$. p . pallens may very well be the hybrid form between the two original populations (Barr 1967). Confirmatory cytological evidence for a very close systematic relationship among them comes from the work of Kanda (1968) who has shown that Japanese populations of fatigans and pallens are karyotypically similar to each other. In the light of these observations, some of the more recent developments in the field of genetic manipulation

1) Text of a paper presented at a working conference on schistosomiasis and filariasis, sponsored by the U.S. and Japanese Panels on Parasitic Diseases of the U.S.-Japan Cooperative Medical Science Program and held in Tokyo during 7-11 November, 1970. The work of the author included herein received partial support from U.S. Atomic Energy Commission Contract (11-1) 38 with the Radiation Laboratory, University of Notre Dame. This is AEC Document No. COO-38-766. 
of Culex pipiens in several parts of the world could be applicable to members of the $C$. pipiens complex that transmit filariasis in Japan. Concerning Aedes togoi, the other major filariasis vector in Japan, some of the methods suggested for Aedes aegypti control (WHO 1968; Craig 1968; Rai 1968; Knipling et al. 1968) could be considered for application to $A$. togoi also.

It is well known that a combined parasitological control of Wucheraria bancrofti infectious in human populations and the vector control in the endemic regions is essential for any long lasting and permanent filariasis control (WHO 1962). All too often, major emphasis is placed on parasitological control. Vector control methods are either not employed at all (e.g., in Brazil, Rai 1969a) or are ineffective because of well known reasons. The end result is that often after several years of campaigns, a significant decline in the incidence of filariasis does not ensue. This was shown to be the case in Ceylon between the years 1959-1964 (Sasa 1965) and in Brazil during the years 1960-68. (Rai 1969), to use two examples. Of course, in properly organized antifilariasis campaigns, it has been and will be possible to depress the infection rates and vector populations. using traditional means of parasite and vector control. It is extremely doubtful however, if these could be completely eradicated by these methods. It is for these reasons that a considerable interest currently exists in the development and field testing of the more efficient methods of vector control.

\section{POSSIBLE GENETIC METHODS FOR CONTROL OF FILARIASIS VECTORS AND THEIR STATUS}

This paper reviews the work done on the development and, in a few cases, on field testing of the following genetic methods which may be applicable for control of filariasis. vectors in Japan:

\section{Sterile male technique}

The status of the sterile male technique for mosquito control has been recently reviewed in detail (Rai 1969b) and the same will therefore not be repeated here. This technique is based on the induction of sexual sterility in males through the use of radiation or chemical sterilants and on flooding natural populations with such males (Knipling 1959). In the case of $C$. fatigans, the first field trial involving releases of radiation-sterilized males was conducted in India (Krishnamurthy et al. 1962). However, this experiment was prematurely terminated because of objections from the villagers at the site after releases of a rather small number of 24000 sterile males over a short period of 35 days. Although a reduction of $6 \%$ in hatchability of egg rafts in this experiment, compared with the control, indicated that matings between the sterile males released and native females did take place, the results were at best inconclusive.

Recently, Patterson et al. (1970) have reported complete suppression of an indigenous population of $C$. p. quinquefasciatus from a small island off the coast of Florida following releases of $8400-18000$ chemosterilized males per day of this species for six genera- 
tions in a 10 -week period. The mosquitoes were sterilized in the pupal stage by exposing them for four hours to an aqueous solution of 0.75 percent thiotepa (tris (1-aziridinyl) phosphine sulfide). A wide safety margin exists between sterilizing and toxic dose levels with this procedure.

Obviously, this successful demonstration indicating that the sterile male technique can be successfully used to eliminate the very important mosquito vector of filariasis from an isolated locality may be regarded as a most significant development following several earlier and unsuccessful attempts with this and other mosquito species in early 1960's.

Coupled with this successful demonstration, the following favorable developments indicate that in not too distant a future, the sterile male technique could be applied for operational vector control:

a. Brown (1967) has reported the development of traps to which male mosquitoes could be attracted either through light or specific baits. This provides a means for autochemosterilization and represents an important advance. Using a battery-operated CDC trap fitted with UV light, Culex fatigans adults were drawn into a chamber treated with tepa. The escaping adults showed $87-93 \%$ sterility. If large numbers of adult mosquitoes can be thus sterilized and dispersed in nature, several problems would be solved simultaneously. Furthermore, autochemosterilization of females along with males would provide an additional advantage. Nevertheless, additional research is needed to ensure that adults thus treated will not carry chemosterilant residues on their bodies and thus become hazardous.

b. Based on an evaluation of the possible causes responsible for earlier and unsuccessful attempts with the sterile male technique, it has been concluded that induction of 100 percent sterility which may often adversely affect male competitiveness is not essential. Thus, treatments which will induce 95-99 percent sterility may prove more effective in achiving control.

\section{Cytoplasmic incompatibility}

Historically, the use of genetic incompatibility among allopatric populations of $C$. pipiens as a means for the control of this species (Laven 1967) has generated more interest in genetic methods of mosquito control than any other single technique. Laven (1967) field tested this method in a small isolated village, Okpo, near Rangoon, Burma and reported "eradication" of $C$. p. fatigans in approximately 3 months or five to six generations following releases of an incompatible male strain. Similar incompatibility in Aedes scutellaris species complex could also be used for genetic control (Knipling et al. 1968).

\section{Interspecific incompatibility}

Interspecific incompatibility Detween Aeaes poıynesıensıs temales, a major vector of filariasis in Polynesia, and $A$. albopictus males has been suggested as a means for controlling the former species. A. albopictus males readily inseminate A. polynesiensis females but the eggs are infertile. Gubler (1970) has reported eradication of a cage colony of $A$. polynesiensis by the addition of $A$. albopictus males at a ratio of 10 albopictus to 1 polynesiensis males. 


\section{Chromosomal translocations}

One possible genetic control method that has attracted considerable attention during: the last few years is the use of inherited sterility associated with chromosomal translocations. The use of this method to control mosquito populations has been proposed by Rai (1967), Rai and Asman (1968), and Rai and McDonald (1970) for Aedes aegypti and by Laven (1969) for Culex pipiens.

The use of translocations for population control may provide some advantages over the sterile male technique. Sterile males do not transmit sterility to members of the next generation and, therefore, continued reintroductions are essential to maintain control. On the contrary, Curtis (1968a) has calculated that a single release of translocation homozygotes of both sexes will reduce an idealized tsetse fly population to zero in about 12 generations; whereas releases of sterile males of equal numbers cannot do that. Furthermore, an additional advantage for the translocation system may accrue from the facts that no physical treatment is given the released insects in the generation in which they are released and that the translocation systems are usually associated with an essentially unaffected genotype and phenotype. As a result, the competitive ability of translocated insects may not be hampered.

Laven (1969) and Rai and McDonald (1970) have reported results of population cage experiments with $C$. pipiens and $A$. aegypti respectively in which the feasibility of the use of males heterozygous for a single translocation was evaluated. In our work, when such males were tested against males from a control stock $(R E D)$ in proportions of $1: 1$, $2: 1,4: 1,6: 1$ or $13: 1$ for matings with $R E D$ females, the translocated males were either equally competitive or often outcompeted normal males and thus in subsequent generations produced expected or more than expected translocated progeny respectively. Laven (1969) also observed an efficiency index of male-linked translocations in Culex pipiens ranging between 1.7-2.1 compared with the efficiency index of 1 for normal males. These results indicate that with proper manipulations, translocations can be used for mosquito control.

At Notre Dame, we are interested in evaluating the potential of sex-linked versus autosomal translocations for genetic control purposes. For each sex-linked translocation, the following two types of translocation heterozygous males have been constructed (Rai et al. 1970):

a. Males in which the male-determining chromosome is translocated $\left(T_{M}\right)$.

b. Males in which the female-determining chromosome is translocated $\left(T_{m}\right)$.

Also, by crossing females heterozygous for one translocation with males heterozygous for another, it was possible to construct males heterozygous for two translocations whose fertility is theoretically one half the fertilty of each translocation heterozygote or 25 percent of a control stock, though in practice, such double heterozygotes were only 12.5 percent fertile (MacDonald and Rai 1970a).

On theoretical grounds, it is expected that the rate of population suppression following releases of males in which the female chromosome is translocated $\left(T_{m}\right)$ should be more rapid than comparable releases of males in which the male-determining chromosome is translocated $\left(T_{M}\right)$. This happens because in the former case female translocation heterozygotes will be produced among the progeny which will themselves be 
semisterile. Furthermore, when such females mate with males heterozygous for the same translocation, translocation homozygotes will be produced and if these homozygotes are inviable, the overall population will be proportionately reduced. The most rapid rate of population decline is expected to ensue from releases of males heterozygous for two translocations. These expectations have been borne out by the computer simulation programs undertaken in our laboratory for Aedes aegypti. Such computer simulation programs based on different patterns of releases of translocated males into idealized populations have shown that if six introductions of four times as many translocated males as are present in a natural population are made, it takes eleven, six and five generations to "eradicate" a population if $T_{M}, T_{m}$ or double translocation heterozygotes respectively are released. These figures were based on the assumption that the idealized population is stable. It takes six generations to "eradicate" a population undergoing a fivefold increase in each generation through the releases of $4: 1$ double heterozygotes (McDonald and Rai 1970b).

Since several sex-linked translocations are available in C. pipiens (Laven 1969), the above conclusions should apply equally well for $C$. p. pallens in Japan and to Aedes togoi when and if translocations are induced in this species.

Unfortunately, it has not yet been possible to obtain translocation homozygotes for any mosquito species. A greatly improved potential for genetic control would ensue when such homozygotes are recovered. In addition to their population suppression potential, Curtis (1968b) has proposed a possible application of the use of translocation homozygotes to fix desirable genes in insect populations. If such homozygotes (TT), carrying genes for refractoriness to diseases or genes conferring insecticide susceptibility are released into populations in numbers "so that the $\mathrm{T}$ frequency in the wild population exceeded the equilibrium point, the population would gradually approach fixation both for the $\mathrm{T}$ chromosomes and the gene for non-infectibility". Thus the genetic structure of a vector population could be changed such that a population becomes unable to transmit a disease. A single gene is already known which determines whether a population of the mosquito, Aedes aegypti, will be susceptible or refractory to Brugia malayi development (MacDonald and Ramachandran 1965). Similar genetic differences in Culex fatigans populations undoubtedly exist and can be manipulated through the use of translocation homozygotes.

\section{Lethal factors}

In addition to the above mechanisms, there are several other methods which have been discussed by Knipling et al. (1968) and some of which could conceivably be applied for control of filariasis vectors in Japan. The use of lethal factors in particular may be pointed out. Barr and coworkers have isolated and described several genetic mutants in Culex pipiens and have suggested the use of recessive lethal genes for genetic control purposes (Barr and Meyers 1966). A female lethal factor is of particular interest since it expresses itself only in females (Barr unpublished). As a result, all or almost all female progenies die, while the males remain unaffected. Such a factor in combination with male-linked translocations of the type described by Laven (1967) and Rai et al. (1970) could be used very effectively to spread simultaneously sterility through 
males and lethality through females.

\section{INTERNATIONAL AGENCIES AND FILARIASIS CONTROL}

Because of its importance in public health, the World Health Organization (WHO) and the International Atomic Energy Agency (IAEA) are particularly interested in evaluating the feasibility of filariasis control through any of the above mentioned genetic methods in several countries. In response to requests from individual governments, IAEA has undertaken such feasibility studies for the application of sterile male technique for filariasis control in Ceylon (Rai 1966) and Brazil (Rai 1969a). Pilot field trials are expected to be undertaken in these countries in the near future.

The World Health Organization has been instrumental in sponsoring extensive research in its field units; e. g., Filariasis Research Unit, Rangoon, Burma, where, as already mentioned, the first positive demonstration of the use of any genetic technique for eradicating Culex fatigans in a small village was made (Laven 1967). In addition, WHO has initiated a Field Unit in Delhi on "Genetic Control of Culicine Mosquitoes" for a six year period (1970-1975) and where one or more of the above mentioned techniques will be evaluated for operational filariasis control on a scale much larger than has been the case to date in any part of the world.

\section{CONCLUSIONS AND SUMMARY}

Although the sterile male method had a somewhat uncertain and discouraging start because of three unsuccessful attempts with this technique, recent positive demonstrations in Okpo, Burma and Seahorse Key, Florida, U. S. A. may be regarded as major breakthroughs which have demonstrated that with proper manipulations, it may be possible to genetically control, perhaps eradicate major vectors of filariasis. The following developments and/or attributes of mosquito distribution tend to reaffirm the above prognosis:

a. Since the male mosquitoes in general emerge before females, previously it was often feared that emerging females may be inseminated by their own sibs as they emerge from their breeding sites. This fear was compounded by the demonstration that multiple inseminations do not take place in mosquitoes and that the sperm from the first mating are used throughout the reproductive life of a female (Craig 1967). However, Gwadz and Craig (1968) have shown that Aedes aegypti females are not inseminatnd unless they are approximately 2 days old. The exact duration of this period depends upon the environment and the strain used. It appears that such a delayed female sexual receptivity is characteristic of mosquitoes in general. This greatly enhances the possibility of the application of the sterile male and other genetic techniques for mosquito control.

b. Several mosquito species which transmit important human diseases, such as filariasis occasionally occur as localized, highly isolated populations. Good examples are provided by a discontinuous distribution of filariasis in Brazil (Rai 1969a) and in small island-like situations with Culex fatigans populations surrounded by large bodies of clear water as in Ceylon (Rai 1966). I suspect the situation in Japan may be similar. The density of these populations can be greatly reduced by traditional methods of 
chemical control and by elimination of artificial breeding sites. After such populations are thus suppressed, inundating these with sterilized, translocated or incompatible males may be entirely feasible.

c. Economical mass production techniques which are crucial for genetic control have been already developed for certain species of mosquitoes (Smith 1967). Facilities to produce 2 million Aedes aegypti per week currently exist. Gerberg et al. (1969) have reported procedure for mass rearing of Culex pipiens.

Nevertheless, the best that can be said as of today concerning filariasis control through genetic manipulation is that although there is "light" at the end of the "tunnel", the tunnel itself is long and narrow and has just not been trodden before. In particular, the paucity of reliable information dealing with several important aspects of behavior of mosquito populations particularly under field conditions cannot be over emphasized. We know relatively little about flight range and adult dispersal; where, when and under what conditions matings take place and the absolute size of natural populations. Thus more research on the ecology and dynamics of field populations is urgently needed before the feasibility of genetic control on a relatively large scale can be effectively evaluated. Furthermore, properly executed experimental field trials on a relatively large scale are needed and should be undertaken in the near future. As emphasized by Rai (1969b) "doubtlessly the groundwork must be prepared with extreme care and precision, and additional data on the biology of those populations where ultimately field trials might be contemplated must be collected and evaluated before actual releases. The selection of a proper site for field evaluation is of utmost importance and if possible island-like experimental sites should be located".

Finally, in order to achieve their maximum potential, genetic methods should be used only as adjuncts to traditional methods of filariasis control. In summary, it may be mentioned, that although the above methods have been discussed in the context of Japanese filariasis vectors, they should be applicable in other countries as well, provided the vector species are similar.

\section{LITERATURE CITED}

Barr, A. R., 1967 Occurrence and distribution of the Culex pipiens complex. Bull. Wld. Hlth. Org. 37: 293-296.

Barr, A. R., and C. M. Meyers, 1966 Inheritance of a lethal affecting larval antennae in Culex pipiens L. (Diptera: Culicidae). J. Parasit. 52: 1163-1166.

Bekku, H., 1956 Studies on the Culex pipiens group of Japan. Nagasaki Med. J. 31: 956-966.

Brown, A. W. A, 1967 Control of Culex fatigans by chemosterilization. WHO Coordination Group on Genetic Control of Insects of Public Health Importance. VBC/CGGC/10.67.

Craig, G. B., Jr., 1967 Mosquitoes: Female monogamy induced by male accessory gland substance. Science 156: 1499-1501.

Craig, G. B., Jr., 1968 Genetic control of Aedes mosquitoes: Current status. Proc. Internat. Congr. Trop. Med. Malar. 8: $975-976$ (abst.).

Curtis, C. F., 1968a A possible genetic method for the control of insect pests, with special reference to tsetse flies (Glossina spp.). Bull. Entomol. Res. 57: 509-523.

Curtis, C. F., 1968b Possible use of translocations to fix desirable genes in insect pest populations. Nature 218: 368-369.

Gerberg, E. J., T. M. Hopkins, and J. W. Gentry, 1969 Mass rearing of Culex pipiens L. Mosquito News 29: 382-385. 
Gubler, D. J., 1970 Induced sterility in Aedes (Stegomyia) polynesiensis Marks by cross-insemination with Aedes (Stegomyia) albopictus Skuse. J. Med. Ent. 7: 65-70.

Gwadz, R., and G. B., Craig, Jr., 1968 Sexual receptivity in female Aedes aegypti. Mosquito News 28: $586-593$.

Kanda, T., 1968 Studies on karyotypes of some Japanese mosquitoes. Japan. J. Exp. Med. 38: $37-46$.

Knipling, E. F., 1959 Sterile-male method of population control. Science 130: 902-904.

Knipling, E. F., H. Laven, G. B. Craig, Jr., R. Pal, J. B. Kitzmiller, C. N. Smith, and A. W. A. Brown, 1968 Genetic control of insects of public health importance. Bull. Wld. Hlth. Org. 38: $421-438$.

Krishnamurthy, B. S., S. N. Ray, and G. C. Joshi, 1962 A note on preliminary field studies of the use of irradiated males for reduction of $C$. fatigans Wied. populations. Ind. J. Malar. 16: $365-373$.

Laven, H., 1967 Eradication of Culex pipiens fatigans through cytoplasmic incompatibility. Nature 216: 383-384.

Laven, H., 1969 Eradicating mosquitoes using translocations. Nature 221: 958-959.

MacDonald, W. W., and C. P. Ramachandran, 1965 The influence of the gene $f^{m}$ (filarial susceptibility, Brugia malayi) on the susceptibility of Aedes aegypti to seven strains of Brugia, Wucheraria and Dirofilaria. Ann. Trop. Med. \& Parasit. 59: 64-73.

McDonald, P. T., and K. S. Rai, 1970a Aedes aegypti: Origin of a "new" chromosome from a double translocation heterozygote. Science 168: 1229-1230.

McDonald, P. T., and K. S. Rai, 1970b Population control potential of heterozygous translocations as determined by computer simulations. Bull. Wld. Hlth. Org. (In press).

Patterson, R. S., D. E. Weidhaas, H. R. Ford, and C. S. Lofgren, 1970 Suppression and elimination of an island population of Culex pipiens quinquefasciatus with sterile males. Science 168: $1368-1369$.

Rai, K. S., 1966 Feasibility study on the application of the sterile male technique for the control of the filariasis vector, Culex fatigans, in Ceylon. International Atomic Energy Agency, Vienna, special publication WP/5/283: $38 \mathrm{pp}$.

Rai, K. S., 1967 Manipulation of cytogenetic mechanisms for genetic control of vectors. WHO Scientific Group on the Cytogenetics of Vectors of Disease of Man. SC/VG. 67. 34, 12 pp.

Rai, K. S., 1968 Genetic control of mosquitoes. Proc. XIII Internat. Cong. Ent. Moscow, U.S.S.R. (In press).

Rai, K. S., 1969a "Isotopes in Entomology: Report to the Government of Brazil". Internat. Atomic Energy Agency Special Publication, Vienna, Austria, WP/5/483, 38 pp.

Rai, K. S., 1969b The status of the sterile male technique for mosquito control. 102-114. In: "Sterile Male Technique for Eradication or Control of Harmful Insects", Doyle, E., Shelagh, Freeman, M. eds. Internat. Atomic Energy Agency, Vienna.

Rai, K. S., and Sr. M. Asman, 1968 Possible application of a reciprocal translocation for genetic control of the mosquito, Aedes aegypti. Proc. XII Internat. Cong. Genetics, Tokyo, Japan: Vol. 1: 164 (Abst.).

Rai, K. S., and P. T. McDonald, 1970 Chromosomal translocations and genetic control of Aedes aegypti. In: "The Sterility Principle for Insect Control or Eradication", LaChance, L. editor. International Atomic Energy Agency, Vienna.

Rai, K. S., P. T. McDonald, and Sr. M. Asman, 1970 Cytogenetics of two radiation-induced, sexlinked translocations in the yellow-fever mosquito, Aedes aegypti. Genetics 66: 35-651.

Sasa, M., 1965 WHO Assignment Report on filariasis control in Ceylon. (TA/56), 18 pp.

Smith, C. A., 1967 "Mass breeding procedures". 653-672 In: Genetics of Insect Vectors of Disease, Wright, J. W., and Pal, R., eds. Elsevier, Amsterdam.

World Health Organization. 1962 Report of Expert Committee on Filariasis. Wld. Hlth. Org. Tech. Rep. Ser. 233: 49 pp., Geneva.

World Health Organization. 1968 Cytogenetics of Vectors of Disease of Man. Wld H1th Org. Tech. Rep. Ser. 398: 41 pp., Geneva. 